\title{
Exosomes for HIV treatment: some advances and perspectives
}

\author{
Tiago Degani Veit ${ }^{1,2 \#}$, Joel Henrique Ellwanger ${ }^{1,3 \#} \wedge$, José Artur Bogo Chies ${ }^{1,3} \wedge$
}

${ }^{1}$ Laboratory of Immunobiology and Immunogenetics, Department of Genetics, Universidade Federal do Rio Grande do Sul (UFRGS), Porto Alegre, Rio Grande do Sul, Brazil; ${ }^{2}$ Institute of Basic Health Sciences, Department of Microbiology, Immunology and Parasitology, Universidade Federal do Rio Grande do Sul (UFRGS), Porto Alegre, Rio Grande do Sul, Brazil; ${ }^{3}$ Postgraduate Program in Genetics and Molecular Biology (PPGBM), Department of Genetics, Universidade Federal do Rio Grande do Sul (UFRGS), Porto Alegre, Rio Grande do Sul, Brazil

\#These authors contributed equally to this work.

Correspondence to: Dr. José Artur Bogo Chies. Laboratório de Imunobiologia e Imunogenética (Prédio 43323, Laboratório 212), Departamento de Genética, Instituto de Biociências, Universidade Federal do Rio Grande do Sul (UFRGS), Av. Bento Gonçalves 9500, Campus do Vale, Porto Alegre, Rio Grande do Sul, CEP 91501-970, Brazil. Email: jabchies@terra.com.br.

Comment on: Shrivastava S, Ray RM, Holguin L, et al. Exosome-mediated stable epigenetic repression of HIV-1. Nat Commun 2021;12:5541.

Received: 07 December 2021; Accepted: 16 February 2022; Published: 28 February 2022.

doi: $10.21037 /$ exrna-21-32

View this article at: https://dx.doi.org/10.21037/exrna-21-32

Different cell types release many classes of extracellular vesicles (called exosomes, microvesicles, microparticles, ectosomes, among others) in biological fluids, which participate in numerous physiological and pathological processes. The understanding and interest in extracellular vesicles have grown significantly in the last decade, and the therapeutic potential of these membranous structures is still being unraveled (1). Among extracellular vesicles, exosomes are the most known and studied subgroup, currently also called "small extracellular vesicles". Exosomes are extracellular vesicles with a lipid bilayer membrane and associated proteins, having an average size of $\sim 100$ nanometers in diameter, and an endosomal origin. Exosomes can package and transport biological cargoes (different types of RNAs, DNA, lipids, metabolites, proteins) between various body compartments, mediating intercellular communication. These nanovesicles can also act by removing excessive or unnecessary molecules from cells. Exosomes participate in immune responses, pregnancy, and modulate the development of different diseases, including central nervous system-related conditions, cancer, cardiovascular diseases and viral infections. Drugs, immune modulators and other biological molecules can be artificially incorporated into exosomes for the treatment of different diseases through the regulated exosome-mediated delivery of these therapeutic components (2).

Interactions between exosomes and viruses are observed in multiple biological contexts. For example, exosomes participate in inflammation, immunomodulation and coagulation during the course of infection with severe acute respiratory syndrome coronavirus 2 (SARS-CoV-2) in humans (3). Exosomes derived from cells infected with human T-lymphotropic virus type 1 (HTLV-1) contain proinflammatory mediators, Tax protein, and other viral components, modulating HTLV-1 pathogenesis and disease progression (4). During pregnancy, the "cloud of exosomes" present at the maternal-fetal interface (5) could influence the risk of transmission of viruses from the mother to the fetus (6). Similarly, seminal exosomes could affect the risk of infection by sexually transmitted viruses (6). Also, exosomes potentially mediate the transmission of human hepatitis C virus (HCV) (7), Zika virus (8), and, as previously mentioned, SARS-CoV-2 (3).

Almost twenty years ago, Gould et al. (9) published the "Trojan exosome hypothesis", arguing that retroviruses, including HIV, could exploit the host's exosome machinery for biogenesis of viral particles and infection, in a mode of infection independent of interaction between the viral envelope protein and host cell receptor. Such publication has shed light on the potential interactions between

^ ORCID: Tiago Degani Veit, 0000-0002-7390-4583; Joel Henrique Ellwanger, 0000-0002-1040-2738; José Artur Bogo Chies, 0000-0001$7025-0660$. 
exosomes and HIV and much work investigating such interactions has since been carried out, adding complexity to this topic. Currently, multiple types of interactions between HIV and exosomes are known, once these vesicles mediate the immune regulation and pathogenesis of HIV infection $(10,11)$. In some situations, exosomes could favor the progression of HIV infection. On the other hand, exosomes could act as fundamental pieces of the immune system for the control of HIV infection [for detailed discussion, see Ellwanger et al. (12)]. As mentioned above, exosomes can be artificially manipulated by incorporating microRNAs or immunoregulatory molecules inside exosomes, for example. Therefore, the potential use of manipulated (or "engineered") exosomes for therapeutic strategies $(13,14)$, including the treatment of SARS-CoV2-related complications (15) and HIV infection (16), is an emerging and promising topic.

Currently, HIV infection is a clinical condition easily manageable through the use of combined antiretroviral therapy (ART). Modern ART regimens are highly effective in sustaining viral load suppression, reducing morbidity, and prolonging the survival of $\mathrm{HIV}$-infected individuals. Moreover, ART prevents HIV transmission, showing that anti-HIV therapy benefits both infected and non-infected individuals. Current ART causes significantly fewer side effects than past generations of ART regimens. However, drug resistance is a significant concern in the treatment of HIV infection and the use of ART still can cause adverse effects in a portion of individuals (17), and therefore the search for new a generation of anti-HIV therapies is welcome.

After infecting host cells, HIV can remain latent for long periods in the infected individual as proviral DNA, integrated into the host genome. Researchers have been trying different strategies to deal with this issue. One way of dealing with HIV latent reservoirs is to "shock and kill", e.g., reversing HIV latency with latency-reversing agents such as histone deacetylase inhibitors, and then killing the infected cells. Another way of dealing with HIV latency is trying to render this latent state permanently, thus blocking the formation of new viral particles in the infected individual. Some latency-inducing strategies have been developed to promote latency but led to either viral resistance and/or toxic side effects $(18,19)$.

The HIV 5'-long terminal repeat (LTR) exerts greater control of viral transcription which could be targeted by strategies aimed at inducing HIV long-term latency. In the pursuit of such an effect, Shrivastava et al. (20) recently developed a recombinant protein system aimed at silencing integrated HIV by inducing DNA CpG methylation specifically at the HIV 5'-LTR. Remarkably, in this proofof-concept study, exosomes were used as a delivery system. To do this, they first designed a gene construct (named ZPAMt) that included active domains of DNA methyl transferase 3 alpha (DNMT3A) fused to HIV-1 promotertargeting zinc finger protein (ZFP-362, which specifically targets position 362 of the HIV 5'-LTR) and a nuclear localization sequence. This construct system was transfected into CHI-Ju cells (Jurkat cells stably infected with HIV-1) and proved to stably repress HIV expression in a sitespecific manner, being resistant to the action of various latency reactivation agents. In order to get this recombinant system to be packaged into exosomes, the authors made use of an already available exosome packaging system called EXOtic (exosome packaging into cells) (21). This system consists of three plasmids encoding (I) exosome proteins associated with increased exosome secretion; (II) the CD63 protein, a marker of exosomes, fused with to L7Ae, an archaebacterial-derived peptide which allows encapsulation into exosomes of RNA sequences that contain a $\mathrm{C} / \mathrm{D}_{\text {box }}$; and (III) a muted Connexin43 (S368A) which associates to form pores at the exosomal membrane allowing for the transfer of cargo into target cells. In order to get ZPAMt to be incorporated into exosomes, a $C / \mathrm{D}_{\mathrm{box}}$ sequence was added to the plasmid containing ZPAMt. Then the authors (20) transfected HEK-293T cells with all four plasmids in order to generate exosomes packed with the gene silencing construct. Despite the relatively low packing of ZPAMt mRNA into exosomes (7,000 copies per $10^{8}$ exosomes), exosomes containing the ZPAMt repressor system were effective in suppressing HIV-1 expression in CHI-Ju cells (HIV-1-chronically infected Jurkat cells) and human peripheral blood mononuclear cells (PBMCs).

In order to test the effect of ZPAMt-containing exosomes in vivo, NSG mice were engrafted with HIV1 -infected PBMCs and then subjected to combined ART for 2 weeks, which was followed by weekly doses of $10^{11}$ exosomes. After 10 weeks following PBMC engraftment, these hu-PBMC-NSG mice treated with ZPAMt exosomes showed a significant decline of HIV RNA copies in the bone marrow and spleen as compared to those treated with control exosomes. This effect of the therapeutic exosome therapy in controlling HIV infection in the brain was analyzed in another mouse model (hu-CD34 ${ }^{+}$NSG) which permits the infection of brain cells by HIV. Again, 10 weeks following infection, a significant reduction in HIV 
RNA was observed in the animals treated for 6 weeks with ZPAMt-packed exosomes, demonstrating that systemically delivered therapeutic exosomes could reach the brain and combat HIV infection in this difficult to reach organ (20). These results demonstrated that engineered exosomes can be used in epigenetics-based therapeutics focused on controlling HIV infection. Also, exosomes have further potential applications for HIV treatment.

Chronic inflammation is a hallmark of HIV infection, occurring in individuals on ART and with different profiles of $\mathrm{HIV}$ infection progression (e.g., rapid progressors, slow progressors), being associated with co-morbidities, including cancer, cardiovascular disease, among others. Multiple factors trigger chronic inflammation in HIV-infected individuals, including residual viral replication $(22,23)$. Therefore, therapies focused on inducing HIV long-term latency, as exemplified above, have the potential to prevent viral replication-associated inflammation. Also, there is some evidence that exosomes contribute to chronic inflammation in HIV infection through the transport of proinflammatory molecules, such as the transcription factor HIF-1 $\alpha$, an inflammation-inducing molecule (22). Therefore, preventing the formation of exosomes containing HIF- $1 \alpha$ could be an interesting therapeutic strategy focused on the reduction of HIV-related chronic inflammation. The packaging of RNA molecules capable of repressing inflammatory molecules within exosomes and subsequent systemic infusion into HIV-infected individuals also has the potential to be used to reduce chronic inflammation in these individuals.

Nevertheless, some points still deserve attention before safely applying exosome-based anti-HIV therapeutics in clinical practice. Firstly, a long-term follow-up would be needed in order to determine the actual consequences of engineered exosomes on the immune system as a whole. One main issue resides on whether such a treatment could negatively influence cell functioning, e.g., affecting proliferation potential or the capacity to quickly mount a given immune response. Although Shrivastava et al. (20) claim this as specific hypermethylation of an integrated provirus as demonstrated by no perceived off-target effect in DNA methylation of the human GAPDH promoter, this specificity and absence of side effects should also be demonstrated in vivo, considering long-term treatments, and upon challenging the immune system with a variety of immunogens. Taking into consideration that such treatment could be used to control the proinflammatory state present in ART-treated individuals, a balance should be achieved between maintenance of a finely tuned immunocompetent system while avoiding immunocompromised states such as immune exhaustion or anergy. Also, determining dosage as well the optimal methodological procedures are significant challenges. In the mouse model, six exosome doses injected weekly were required in order to achieve effective epigenetic silencing of HIV-1 and to induce a "block and lock" phenotype (20). Since mice were euthanized not much after the last injection, maintenance of the phenotype for longer periods without the need for further interventions still needs to be demonstrated. Finally, the observed decline in the HIV-1 reservoir in the brain also reminds us about the existence of different cell compartments in a complex organism, with different dynamics of migration and cell interactions that should be evaluated and understood in order to effectively allow the use of such a new approach.

In conclusion, keeping in mind the long road we still need to go to overcome these limitations, the perspectives mentioned above in association with the results reported by Shrivastava et al. (20) support the view of exosomes as promising actors for the advancement of HIV therapy.

\section{Acknowledgments}

Funding: Joel Henrique Ellwanger receives a postdoctoral fellowship from Coordenação de Aperfeiçoamento de Pessoal de Nível Superior [Programa Nacional de PósDoutorado (PNPD)/CAPES, Brazil]. José Artur Bogo Chies receives a research fellowship from $\mathrm{CNPq}$ (Bolsa de Produtividade em Pesquisa-Nível 1A, CNPq, Brazil) and has research project funded by CAPES (CAPES AUXPE 686/2020; Brazil).

\section{Footnote}

Provenance and Peer Review: This article was commissioned by the editorial office, $E x R N A$. The article has undergone external peer review.

Conflicts of Interest: All authors have completed the ICMJE uniform disclosure form (available at https://exrna. amegroups.com/article/view/10.21037/exrna-21-32/coif). JHE receives a postdoctoral fellowship from Coordenação de Aperfeiçoamento de Pessoal de Nível Superior [Programa Nacional de Pós-Doutorado (PNPD)/CAPES, Brazil]. $\mathrm{JABC}$ receives a research fellowship from $\mathrm{CNPq}$ (Bolsa de Produtividade em Pesquisa-Nível 1A, CNPq, Brazil) and has research project funded by CAPES (CAPES AUXPE 686/2020; Brazil). The other author has no conflicts of 
interest to declare.

Ethical Statement: The authors are accountable for all aspects of the work in ensuring that questions related to the accuracy or integrity of any part of the work are appropriately investigated and resolved.

Open Access Statement: This is an Open Access article distributed in accordance with the Creative Commons Attribution-NonCommercial-NoDerivs 4.0 International License (CC BY-NC-ND 4.0), which permits the noncommercial replication and distribution of the article with the strict proviso that no changes or edits are made and the original work is properly cited (including links to both the formal publication through the relevant DOI and the license). See: https://creativecommons.org/licenses/by-nc-nd/4.0/.

\section{References}

1. Théry C, Witwer KW, Aikawa E, et al. Minimal information for studies of extracellular vesicles 2018 (MISEV2018): a position statement of the International Society for Extracellular Vesicles and update of the MISEV2014 guidelines. J Extracell Vesicles 2018;7:1535750.

2. Kalluri R, LeBleu VS. The biology, function, and biomedical applications of exosomes. Science 2020;367:eaau6977.

3. Barberis E, Vanella VV, Falasca M, et al. Circulating Exosomes Are Strongly Involved in SARS-CoV-2 Infection. Front Mol Biosci 2021;8:632290.

4. Jaworski E, Narayanan A, Van Duyne R, et al. Human T-lymphotropic virus type 1 -infected cells secrete exosomes that contain Tax protein. J Biol Chem 2014;289:22284-305.

5. Mincheva-Nilsson L. Placental exosome-mediated immune protection of the fetus: feeling groovy in a cloud of exosomes. Expert Review of Obstetrics \& Gynecology 2010;5:619-34.

6. Kaminski VL, Ellwanger JH, Chies JAB. Extracellular vesicles in host-pathogen interactions and immune regulation - exosomes as emerging actors in the immunological theater of pregnancy. Heliyon 2019;5:e02355.

7. Liu Z, Zhang X, Yu Q, et al. Exosome-associated hepatitis $\mathrm{C}$ virus in cell cultures and patient plasma. Biochem Biophys Res Commun 2014;455:218-22.
8. Zhou W, Woodson M, Sherman MB, et al. Exosomes mediate Zika virus transmission through SMPD3 neutral Sphingomyelinase in cortical neurons. Emerg Microbes Infect 2019;8:307-26.

9. Gould SJ, Booth AM, Hildreth JE. The Trojan exosome hypothesis. Proc Natl Acad Sci U S A 2003;100:10592-7.

10. Ellwanger JH, Crovella S, Dos Reis EC, et al. Exosomes are possibly used as a tool of immune regulation during the dendritic cell-based immune therapy against HIV-I. Med Hypotheses 2016;95:67-70.

11. Sadri Nahand J, Bokharaei-Salim F, Karimzadeh M, et al. MicroRNAs and exosomes: key players in HIV pathogenesis. HIV Med 2020;21:246-78.

12. Ellwanger JH, Veit TD, Chies JAB. Exosomes in HIV infection: A review and critical look. Infect Genet Evol 2017;53:146-54.

13. Kaminski V, Ellwanger JH, Chies JAB. Down-regulation of HLA-G gene expression as an immunogenetic contraceptive therapy. Med Hypotheses 2017;102:146-9.

14. Jafari D, Shajari S, Jafari R, et al. Designer Exosomes: A New Platform for Biotechnology Therapeutics. BioDrugs 2020;34:567-86.

15. Rezakhani L, Kelishadrokhi AF, Soleimanizadeh A, et al. Mesenchymal stem cell (MSC)-derived exosomes as a cellfree therapy for patients Infected with COVID-19: Real opportunities and range of promises. Chem Phys Lipids 2021;234:105009.

16. Ferrantelli F, Manfredi F, Chiozzini C, et al. DNA Vectors Generating Engineered Exosomes Potential CTL Vaccine Candidates Against AIDS, Hepatitis B, and Tumors. Mol Biotechnol 2018;60:773-82.

17. Cihlar T, Fordyce M. Current status and prospects of HIV treatment. Curr Opin Virol 2016;18:50-6.

18. Mousseau G, Aneja R, Clementz MA, et al. Resistance to the Tat Inhibitor Didehydro-Cortistatin A Is Mediated by Heightened Basal HIV-1 Transcription. mBio 2019;10:e01750-18.

19. Vansant G, Bruggemans A, Janssens J, et al. Block-and-lock strategies to cure HIV-1 infection. Viruses 2020;12:84.

20. Shrivastava S, Ray RM, Holguin L, et al. Exosomemediated stable epigenetic repression of HIV-1. Nat Commun 2021;12:5541.

21. Kojima R, Bojar D, Rizzi G, et al. Designer exosomes produced by implanted cells intracerebrally deliver therapeutic cargo for Parkinson's disease treatment. Nat Commun 2018;9:1305.

22. Pérez PS, Romaniuk MA, Duette GA, et al. Extracellular vesicles and chronic inflammation during HIV infection. J 
Extracell Vesicles 2019;8:1687275.

23. Ellwanger JH, Valverde-Villegas JM, Kaminski VL, et al. Increased IL-8 levels in HIV-infected individuals who

doi: 10.21037/exrna-21-32

Cite this article as: Veit TD, Ellwanger JH, Chies JAB. Exosomes for HIV treatment: some advances and perspectives. ExRNA 2022;4:6. initiated ART with CD4+ T cell counts $<350$ cells/mm3 A potential hallmark of chronic inflammation. Microbes Infect 2020;22:474-80. 